\title{
Electro-optical modulating multistack device based on the CMOS-compatible technology of amorphous silicon
}

Sandro Rao

sandro.rao@unirc.it

\section{Francesco G. Della Corte}

\begin{abstract}
Department of Information Science, Mathematics, Electronics and Transportations (DIMET) “Mediterranea" University, Via Graziella Località Feo di Vito, 89131 Reggio Calabria, Italy Department of Information Science, Mathematics, Electronics and Transportations (DIMET) “Mediterranea" University, Via Graziella Località Feo di Vito, 89131 Reggio Calabria, Italy
\end{abstract}

In this paper we report results on a field-effect induced light modulation at $\lambda=1.55 \mu \mathrm{m}$ in a high-index-contrast waveguide based on a multisilicon-on-insulator (MSOI) platform. The device is realized with the hydrogenated amorphous silicon ( $\alpha$-Si: $\mathrm{H})$ technology and it is suitable for monolithic integration in a CMOS Integrated Circuit. The device exploits the free carrier optical absorption electrically induced in the semiconductor core waveguide. The dynamic behaviour of the device was experimentally and theoretically analyzed in presence of a visible illumination showing a link between the photogeneration and the free carriers provided by doped $\alpha$-Si:H layers. The core waveguide contains several thin dielectric films of amorphous silicon carbonitride ( $\alpha$-SiCN) embedded along its thickness highly enhancing the absorbing action of the modulator held in the on-state. [DOI: 10.2971/jeos.2010.10040s]

Keywords: amorphous materials, CMOS integrated circuits, electrooptic modulation, waveguides

\section{INTRODUCTION}

The optoelectronic properties of hydrogenated amorphous silicon, $\alpha-\mathrm{Si}: \mathrm{H}$, and related materials have been exploited for decades almost exclusively for the fabrication of lowcost large-area photovoltaic devices. Recently, thanks to their unique characteristics of transparency at the infrared wavelengths [1], refractive index tunability and good technological compatibility with all microelectronic process [2], many $\alpha$-Si:H-based photonic devices, such as LEDs [3], photodetectors [4] and lightwave guiding [2], have been fabricated successfully. Thermal effects have also been exploited to demonstrate modulation actions in planar devices for integrated photonics [2].

More recently, a $\lambda=1.55 \mu \mathrm{m}$ radiation propagating in a planar waveguide has been modulated in amplitude by a visible wide spectrum light sources [5]. In such cases the interaction of amorphous silicon with an infrared radiation strictly depends on the density of localized states available in the pseudogap and their respective occupancy state. This is a peculiarity of amorphous semiconductors which makes a substantial difference in the optoelectronic applications with respect to the homologous crystalline counterparts.

From this point of view, the possibility of applications of amorphous semiconductors in optoelectronics added a renewed scientific interest to the study of the defect population of the pseudogap, and to its response to external signals such as optical pumping in the visible wavelengths. For these purposes, optical pump and probe measurements, photoconduction or luminescence measurements, or techniques as the photothermal deflection spectroscopy (PDS) [6], are used to obtain fundamental information, such as the concentration and energy distribution of electronic states at the band edges and within the pseudogap.

In contrast, electro-optical effects, have been rarely considered in $\alpha$-Si:H-based devices due to the weak incidence of electric field on the refractive index [7], on one side, and the difficulty of reaching a significant carrier injection across $p-n$ junctions in amorphous semiconductors, on the other side.

In this paper, the recent results $[5,8]$ on all-optical and field effect induced light modulation at $\lambda=1.55 \mu \mathrm{m}$ are further extended with the use of a thinner and more performing $\alpha$ $\mathrm{Si}: \mathrm{H} / \alpha$-SiCN multistack waveguiding device.

The modulation mechanism has been modelled as a free carrier effect on the imaginary part of the refractive index. In particular, the amplitude modulation of the infrared probe beam, launched into the waveguide, is induced through a variation of the effective absorption coefficient. This variation is enhanced, both, by carrier accumulation at the intermediate semiconductor/insulator interfaces and by illuminating, with a visible pump source, above the waveguide. The device speed behaviour was accurately studied by means of dynamic simulations taking into account the free carrier generation (thermal and photoinduced) and trapping phenomena typical of amorphous semiconductors.

Moreover, the carrier profile under bias and illumination was numerically estimated and used for optical simulations. The results are found to be in agreement with the experimental data. 


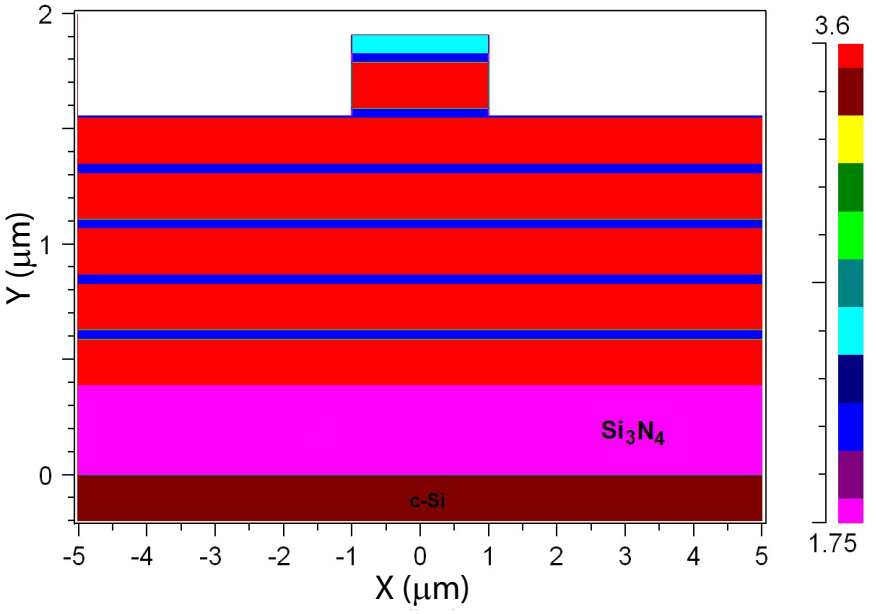

FIG. 1 Schematic cross sections of the realized waveguide and corresponding refractive index colour bar. The crystalline silicon substrate is $300 \mu \mathrm{m}$ thick.

A free-space experimental setup was ad hoc developed in order to control the polarisation state of the input beam (TE or $\mathrm{TM}$ ) entering or exiting the chip under test.

\section{DESIGN AND FABRICATION}

Figure 1 illustrates the schematic cross section considered in this paper. The waveguide consists of the stack of six bilayers each composed of a $0.2 \mu \mathrm{m}$-thick $\alpha$-Si:H layer and a $40 \mathrm{~nm}$-thick $\alpha$-SiCN layer, deposited on a $\langle 100\rangle$ heavily doped silicon substrate. The substrate and the stack are separated by a $0.39 \mu$ m-thick $\mathrm{Si}_{3} \mathrm{~N}_{4}$ low refractive index cladding layer ensuring a strong vertical optical confinement. An $80 \mathrm{~nm}$ thick transparent conductive Indium Tin Oxide (ITO) thin film forms the top contact, while the bottom contact is the Si substrate itself.

The device fabrication process details are illustrated in [8]. The device was optically modelled using Beamprop, the device simulation package from RSoft [9]. The waveguide has been designed in order to ensure only the propagation of the fundamental mode, for this purposes the optical mode spectrum was also modeled. The optical data of the materials used for simulations were determined as reported in [8] and listed in Table 1 together with the PECVD process parameters.

It should be remarked that all of the $\alpha-\mathrm{Si}: \mathrm{H}$ layers are unintentionally $n$-doped, with a free electron concentration of the order of $10^{10} \mathrm{~cm}^{-3}$, and a consequent free hole concentration of $\sim 10^{5} \mathrm{~cm}^{-3}$. For this reason in the following only electrons will be considered in mixed electrical-optical calculations.

Simulation results demonstrated that the designed waveguide supports only one mode for TE polarization once a $2 \mu \mathrm{m}$ wide rib is defined by photolithographic patterning and wet etching in $5 \% \mathrm{HCl}$ solution. The etch depth is $0.35 \mu \mathrm{m}$. Due to the geometry of the waveguide, the TM mode exhibits high losses [10] and low modulation efficiency [11], therefore only the TE-like polarization will be considered hereafter. Figure 2 shows the fundamental $\mathrm{TE}_{0}$ mode, well confined under rib region, characterized by propagation losses of $1.47 \mathrm{~cm}^{-1}$ at

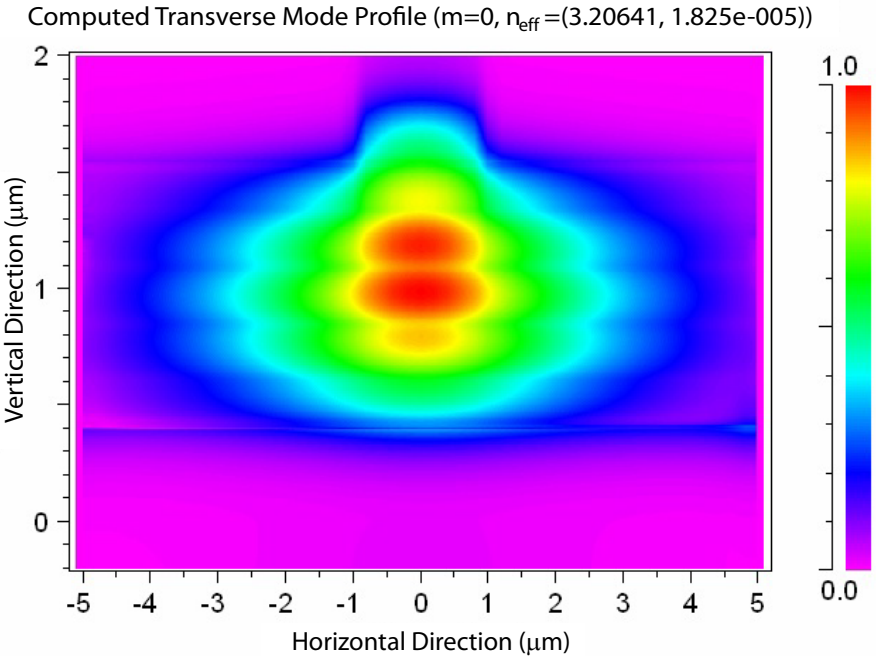

FIG. 2 Fundamental TE mode in the $x$-y plane.

$\lambda=1.55 \mu \mathrm{m}$ in agreement with experimental results. We note, however, that assuming for $\alpha$-Si:H a fundamental absorption as low as $0.1 \mathrm{~cm}^{-1}[1]$, simulations return propagation losses of $0.89 \mathrm{~cm}^{-1}$.

In the presented device the thin $\alpha$-SiCN highly insulating layers [12] break the conduction among the $\alpha$-Si:H films so that the device electrically behaves as the series of capacitors. The application of a biasing voltage between the ITO layer and the c-Si substrate determines an accumulation of electrons and holes at the opposite ends of each internal $\alpha$-SiCN dielectric layer. The presence of $\operatorname{six} \alpha-\mathrm{SiCN}$ regions between the $\alpha$-Si:H layers allows a distribution of the excess free carriers across the device section contributing to create a highly-effective absorption variation. It is clear from this structure that most of the applied electric field falls across the thick $\mathrm{Si}_{3} \mathrm{~N}_{4}$ bottom cladding. An improved design of the device includes a buried bottom contact, made of a thin ITO film laid between the thick $\mathrm{Si}_{3} \mathrm{~N}_{4}$ cladding and the waveguide core. This would in addition release a constraint on the $\mathrm{Si}_{3} \mathrm{~N}_{4}$ thickness, actually set at the present small value for electrical reasons, allowing a thicker cladding for a better optical confinement.

The refractive index and absorption coefficient change in silicon, due to injection or depletion of free carrier at $\lambda=$ $1.55 \mu \mathrm{m}$, can be derived to a first order approximation from the classical Drude model also for amorphous silicon semiconductor [13].

The advantages brought by burying isolating layers in a c-Si core of a waveguide, in order to enhance the modulation efficiency exploiting the free carrier effects, has been proven theoretically by C. A. Barrios [14].

The device was modelled both for its static and dynamic behaviour using ATLAS, the device simulation package from Silvaco [15].

The program simulates internal physics and device characteristics of semiconductor devices by numerically solving Poisson's equation and the charge continuity equations for electrons and holes. In our calculations, a carrier concentration de- 


\begin{tabular}{|c|c|c|c|c|c|c|c|}
\hline Material & $T\left({ }^{\circ} \mathrm{C}\right)$ & Rate $\left(\mathrm{As}^{-1}\right)$ & \multicolumn{2}{|c|}{ Process Gas $(\mathrm{sccm})$} & $n$ & $\alpha\left(\mathrm{cm}^{-1}\right)$ & $\sigma\left(\mathrm{Scm}^{-1}\right)$ \\
\hline $\mathrm{Si}_{3} \mathrm{~N}_{4}$ & 220 & 2.5 & $\begin{array}{c}\mathrm{SiH}_{4} \\
\mathrm{NH}_{3}\end{array}$ & $\begin{array}{c}1.5 \\
68\end{array}$ & $1.77 \pm 0.02$ & $10 \pm 5$ & $<10^{-16}$ \\
\hline$\alpha-\mathrm{Si}: \mathrm{H}$ & 220 & 1.5 & $\mathrm{SiH}_{4}$ & 20 & $3.58 \pm 0.02$ & $1 \pm 0.5$ & $2.6 \times 10^{-9}$ \\
\hline \multirow{2}{*}{$\alpha-\mathrm{SiCN}$} & 220 & 1.62 & $\mathrm{SiH}_{4}$ & 8 & & & \\
& & & $\mathrm{NH}_{3}$ & 24 & $2.03 \pm 0.02$ & $3 \pm 1.5$ & $5.2 \times 10^{-16}$ \\
& & & & & & \\
\hline
\end{tabular}

TABLE 1 PECVD process parameters: substrate temperature, deposition rate. Refractive index $n$ and absorption coefficient $\alpha$ are measured at $1.55 \mu$ m. Conductivity measurements were performed using a computer controlled parametric characterization system based on an automatic probing station.

\begin{tabular}{|c|c|}
\hline$E_{g}(\mathrm{eV})$ & 1.8 \\
\hline$\mu_{n}\left(\mathrm{~cm}^{2} / \mathrm{V} \cdot \mathrm{s}\right)$ & 0.1 \\
\hline$\mu_{p}\left(\mathrm{~cm}^{2} / \mathrm{V} \cdot \mathrm{s}\right)$ & 0.01 \\
\hline$m_{n}^{*}(\mathrm{~kg})$ & $0.34 m_{0}$ \\
\hline$m_{h}^{*}(\mathrm{~kg})$ & $0.34 m_{0}$ \\
\hline$m_{0}(\mathrm{~kg})$ & $9 \times 10^{-31}$ \\
\hline$\chi(\mathrm{eV})$ & 4.1 \\
\hline$E_{G, C}(\mathrm{eV})$ & 0.83 \\
\hline$E_{G, V}(\mathrm{eV})$ & 0.58 \\
\hline
\end{tabular}

TABLE 2 Physical parameters used for the electrical simulations of the $\alpha$-Si:H layer.

pendent Shockley-Read-Hall (SRH) recombination model has been employed, while the simulation parameters were set as reported in Table 2.

Moreover, we considered the carrier trapping effects and the releasing phenomena involving the shallow levels in the disordered $\alpha$-Si:H films [16] by modelling the defects concentration typical of amorphous semiconductors $[17,18]$.

Figure 3 reports the geometrical details (a) of one of the six insulator-silicon-insulator structures forming the device, and the calculated electron concentration depth profile (b).

We obtained a carrier concentration change, close to the interface, of $\sim 10^{18} \mathrm{~cm}^{-3}$ when a bias of $20 \mathrm{~V}$ is applied across the six bi-layer device.

Mixed electrical-optical simulations were run in order to model the modulation effect for the realized device. We calculated, at several bias points, the accumulated carrier profiles and the corresponding free carrier induced optical absorption variations.

The calculated new absorption profile was fed into the optical simulator, which returned a theoretical modulation in good agreement with the measured one. Results are summarized in the following.

For all the configurations, a uniform grid size ( $1 \mathrm{~nm}$ along the vertical axis and $50 \mathrm{~nm}$ along the horizontal direction) was employed for both electrical and optical simulations.
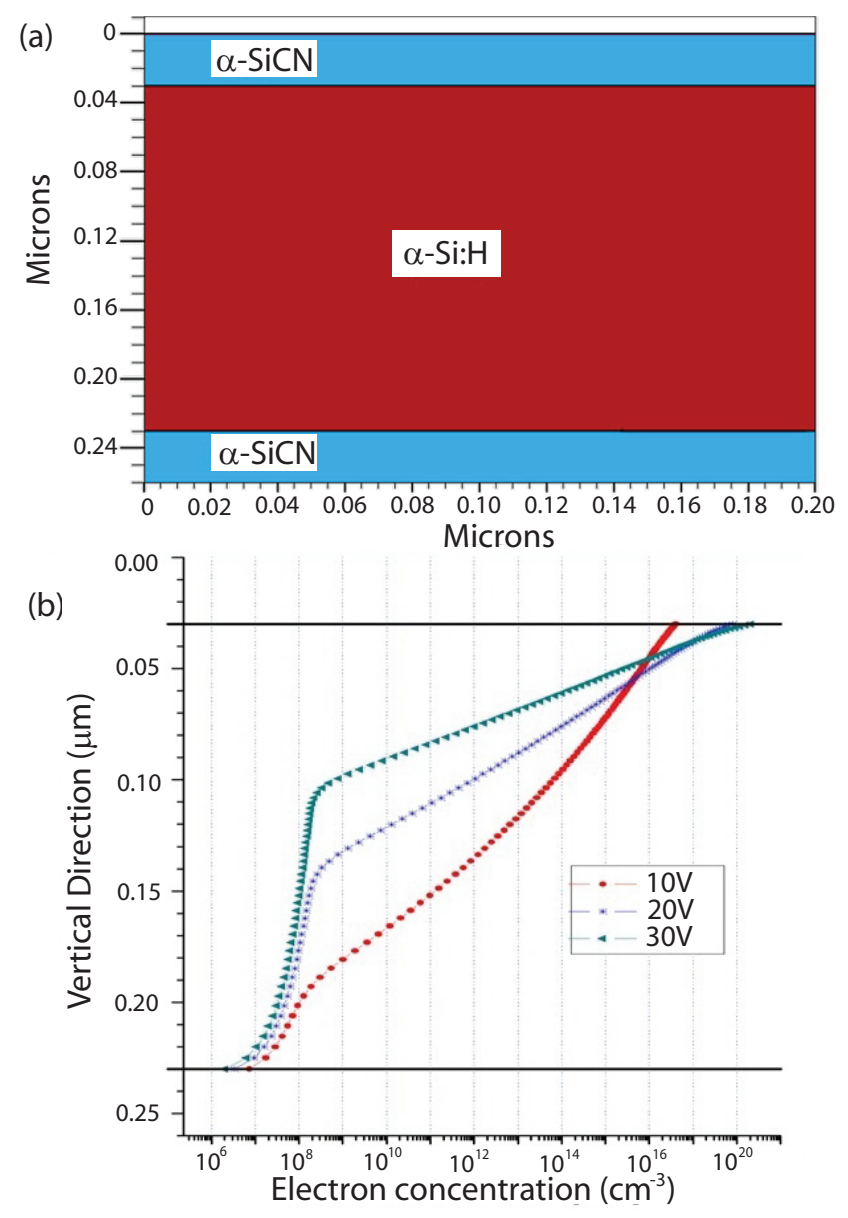

FIC. 3 Estimated electron concentrations $/ \log \left(\mathrm{cm}^{-3}\right)$ (b) along a vertical section positioned at the device center (a). The applied voltages are $10 \mathrm{~V}, 20 \mathrm{~V}$ and $30 \mathrm{~V}$ across the whole six bi-layers structure.

\section{EXPERIMENTAL RESULTS AND DISCUSSION}

\subsection{Modulation measurements}

Modulation measurements were performed avoiding every ambiguity in the polarisation state of an optical signal entering or exiting the chip under test. Hence a free-space experimental setup was developed. A broadband polarising cube beamsplitter and a half-wave plate were placed in the beam path allowing for the linear polarisation state of the input beam to be converted from TE to TM or vice versa. The $15 \mathrm{~mW}$ laser beam, at $\lambda=1.55 \mu \mathrm{m}$, was focused onto the input facet 


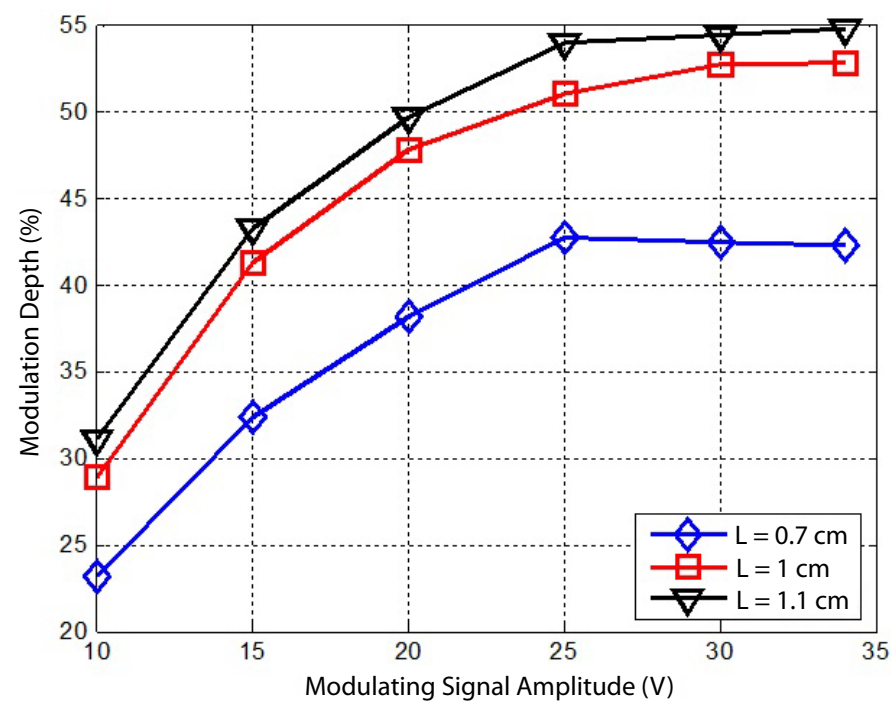

FIG. 4 Dependence of $M_{\%}$ on the modulating signal amplitude for three different lengths ( 6 bi-layers, $1.2 \mu \mathrm{m}$ overall core thickness.

of the waveguide and the modulated light emerging from the chip was collected and collimated by an objective and detected by an InGaAs photodiode.

The waveguide propagation losses were first measured by the cut-back technique [19]. To improve the accuracy of this method, the device was cut from the substrate by cleavage several times. We obtained samples with lengths in the range $0.7 \mathrm{~cm}$ to $1.1 \mathrm{~cm}$. Sample facets did not receive polishing treatment. From these samples we calculated a loss coefficient of $2.26 \pm 0.04 \mathrm{~cm}^{-1}$, mainly due to the fundamental absorption/scattering phenomena in the $\alpha$-Si:H layers (see Table 1) and to the thin $\mathrm{Si}_{3} \mathrm{~N}_{4}$ cladding which does not completely prevent the optical field tail from extending into the lossy substrate. The overall insertion losses of the device are above $11 \mathrm{~dB}$, with the main contribution due to the coupling losses, a drawback of the high refractive index of silicon used as the core material.

Subsequently, a set of three samples $(L=0.7 \mathrm{~cm}, 1 \mathrm{~cm}$ and $1.1 \mathrm{~cm}$ ) were used to characterize the modulation effect by applying square voltage pulses to the electrodes.

For an intensity modulator, where the applied electrical signal acts to decrease the intensity $I$ of the transmitted light, the modulation depth is defined as

$$
M=\frac{I_{\max }-I_{\min }}{I_{\max }}
$$

where $I_{\max }$ and $I_{\min }$ are the maximum and minimum intensity of the transmitted signal.

Figure 4 reports the dependence of $M \%$ on the modulating signal amplitude at a frequency of $20 \mathrm{~Hz}$ and a duty cycle of $50 \%$ for the three different lengths. A maximum modulation depth up to $55 \%$ is obtained for the $1.1 \mathrm{~cm}$ long sample.

The reported data show that $M_{\%}$ increases with the modulating signal amplitude and with the device length. As confirmed by electrical simulations, increasing the external electric field more carriers are accumulated at the opposite sides

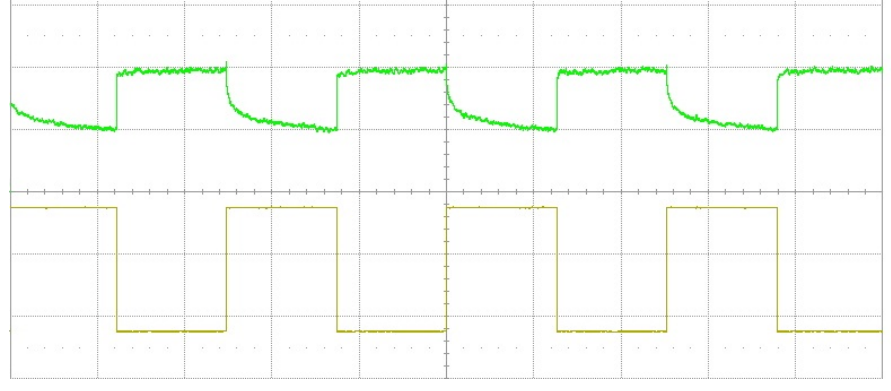

FIG. 5 Applied voltage (bottom trace) and output light power (top trace) for the six bi-layers, $1 \mathrm{~cm}$ long, waveguide. The modulating signal has $V_{\min }=0 \mathrm{~V}, V_{\max }=20 \mathrm{~V}$, duty-cycle $=50 \%$. The time base is $20 \mathrm{~ms} /$ div.

of each insulating film, occupying a larger and larger area of the waveguide where the optical mode propagates.

The average effective absorption coefficient variation $\Delta \alpha_{\text {eff }}$ induced in the core waveguide is $0.35 \mathrm{~cm}^{-1}, 0.65 \mathrm{~cm}^{-1}$ and $0.75 \mathrm{~cm}^{-1}$ respectively at $10 \mathrm{~V}, 20 \mathrm{~V}$ and $30 \mathrm{~V}$ biases, corresponding to an electric field of $0.13 \mathrm{MV} / \mathrm{cm}, 0.26 \mathrm{MV} / \mathrm{cm}$ and $0.39 \mathrm{MV} / \mathrm{cm}$ across the $40 \mathrm{~nm}$ thin insulating $\alpha$-SiCN layers.

Table 3 summarizes the experimental results present in the literature for absorption type modulators based on crystalline silicon, as no other similar devices are known in $\alpha$-Si:H. Our results are also reported for comparison. The first two devices [20, 21] are controlled by bipolar carrier injection. In both cases the high current might be responsible of a heating and a thermal drift. The third device [22] is embedded in a MOS structure, where electric fields as high as $3.6 \mathrm{MV} / \mathrm{cm}$ are reached in the oxide to get $\Delta \alpha_{\text {eff }}=0.4 \mathrm{~cm}^{-1}$.

Figure 5 shows the output light power and the applied voltage as a function of time for one of the considered devices, a $1 \mathrm{~cm}$ long waveguide. The amplitude of the applied pulses voltage is $20 \mathrm{~V}$ and the correspondent calculated modulation depth is $M_{\%}=47 \%$.

In Table 4 we compare the estimated and experimental optical performances of our actual devices. From these data it is evident that the number of bi-layers and their thickness have a strong impact on modulation efficiency. Both approaches in fact increase the relative volume of the waveguide where carrier accumulation takes place leading to a higher interaction between the optical signal and the accumulation layers. For this purpose, a simulation of a multistack structure consisting of nine bi-layers each composed of a $0.1 \mu \mathrm{m}$-thick $\alpha$-Si:H layer and a $30 \mathrm{~nm}$-thick $\alpha$-SiCN layer was performed. Results are also reported in Table 4 .

It is worth noting from Table 4 that the basic simulation set-up underestimates modulation for thinner $\alpha$-Si:H layers, while the contrary applies for thicker devices. However, it should be noted that the agreement is rather good, in spite of the fact that the electrical parameters of $\alpha$-Si:H used in simulations were deliberately taken from the literature [16]-[18].

For the new simulated single-mode waveguide we estimated, from mixed electrical-optical simulations, very high effective 


\begin{tabular}{|c|c|c|c|c|c|}
\hline Structure & Type & $\mathrm{J}\left(\mathrm{kA} / \mathrm{cm}^{2}\right)$ & $\mathrm{E}(\mathrm{MV} / \mathrm{cm})$ & $\Delta \alpha_{\text {eff }}\left(\mathrm{cm}^{-1}\right)$ & Note \\
\hline Reference [21] & Carrier injection & 2 & - & 0.5 & self heating \\
\hline Reference [22] & Carrier injection & 1 & - & 100 & self heating \\
\hline Reference [23] & Field effect & - & 3.6 & 0.4 & \\
\hline Reference [9] & Field effect & - & 0.45 & 0.21 & \\
\hline This work & Field effect & - & 0.39 & 0.75 & \\
\hline
\end{tabular}

TABLE 3 Silicon absorption modulators reported in the literature (PLEASE CHECK AND CORRECT REFERENCES. REFERENCE 23 IS NOT PRESENT IN THE LIST OF REFERENCES).

\begin{tabular}{|c|c|c|c|c|c|c|}
\hline \multirow{2}{*}{ Structure } & \multicolumn{3}{|c|}{$\begin{array}{c}\text { Simulation } \\
\Delta \alpha_{\text {eff }}\left(\mathrm{cm}^{-1}\right)\end{array}$} & \multicolumn{3}{c|}{$\begin{array}{c}\text { Experimental } \\
\Delta \alpha_{\text {eff }}\left(\mathrm{cm}^{-1}\right)\end{array}$} \\
\cline { 2 - 7 } & $\mathbf{1 0 ~ \mathbf { ~ V }}$ & $\mathbf{2 0 ~ V}$ & $\mathbf{3 0 ~ V}$ & $\mathbf{1 0 ~ V}$ & $\mathbf{2 0 ~ V}$ & $\mathbf{3 0 ~ V}$ \\
\hline $\begin{array}{c}\text { Reference [8] } \\
\text { (3 Bi-layers) }\end{array}$ & 0.06 & 0.08 & 0.09 & 0.02 & 0.03 & 0.04 \\
\hline $\begin{array}{c}\text { Reference [8] } \\
\text { (6 Bi-layers) }\end{array}$ & 0.18 & 0.20 & 0.25 & 0.11 & 0.18 & 0.21 \\
\hline This work & 0.27 & 0.52 & 0.68 & 0.35 & 0.65 & 0.75 \\
\hline 9 bi-layers & 0.45 & 2.17 & 3.21 & \multicolumn{4}{|c}{} \\
\hline
\end{tabular}

TABLE 4 Device performance optimization and correspondent experimental effective absorption coefficient variation $\Delta \alpha_{\text {eff }}$.

absorption coefficient variations confirming that it is possible to obtain high modulation depths also with shorter length devices at lower biasing voltages. In particular, a modulation depth of $\sim 80 \%$ could be achieved in a $2 \mathrm{~mm}$-long 9 bi-layers multistack-device by applying an electric field of $0.02 \mathrm{MV} / \mathrm{cm}$ across each $30 \mathrm{~nm}$-thin insulating $\alpha$-SiCN layer.

\subsection{Dynamic Behaviour}

Speed is an important parameter that might be improved by performing a better design. A simulation study of the carrier dynamics taking place in the $\alpha$-Si:H layers reveals that the carrier accumulation phase is actually limited by the thermal generation and trapping phenomena, while the carrier spreading, following the bias removal, is comparatively faster, in agreement with experiments (see Figure 5). Figure 6 shows, for instance, the calculated electron count dynamics at one $\alpha-\mathrm{Si}: \mathrm{H} / \alpha-\mathrm{SiCN}$ interface. One way to circumvent this problem might consist in providing the $\alpha$-Si:H layers with additional free electrons, e.g. by means of an effective doping or by photo-generation.

In order to analyze the dynamic behaviour of the device when external free carriers are provided by photogeneration, in our experiments we used as pump radiation three AlInGaP light emitting diodes (LEDs), all $10 \mathrm{~mm}$ in diameter, at $565 \mathrm{~nm}$, $612 \mathrm{~nm}$, and $660 \mathrm{~nm}$, respectively. The sample surface was illuminated with a homogeneous $16 \mathrm{~mm}^{2}$-wide spot. Measurements at different pump intensities were performed by acting on the bias signal level.

Figure 7 reports the measured modulation depth for the sample under test, $1 \mathrm{~cm}$-long, as a function of the electrical mod-

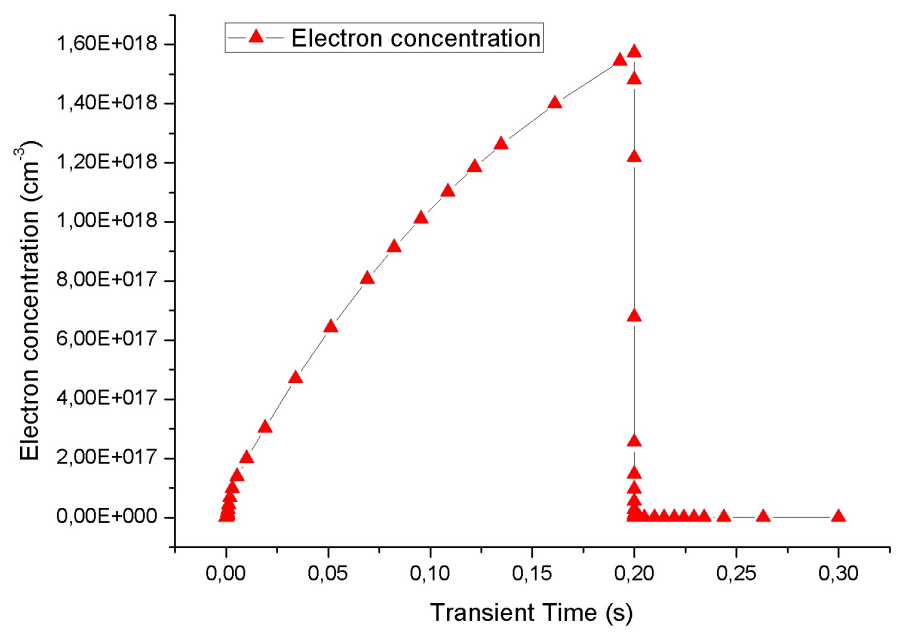

FIG. 6 Accumulated electrons $\left(\mathrm{cm}^{-3}\right)$ at the $\alpha$-Si:H/dielectric interface $(x=0.100, y=$ 0.03 in Figure $3(\mathrm{a})$ ) versus transient time for a modulating signal amplitude of $30 \mathrm{~V}$.

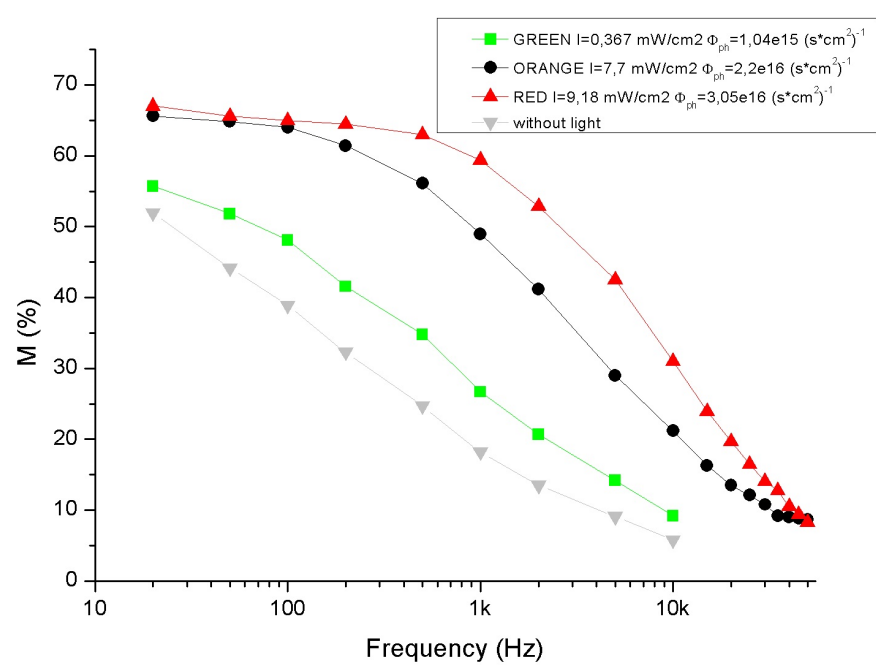

FIC. $7 M_{\%}$ vs. frequency at different pump wavelengths ( $565 \mathrm{~nm}, 612 \mathrm{~nm}$, and $660 \mathrm{~nm}$ ) and powers. The electrical modulating signal has an amplitude of $20 \mathrm{~V}$ and duty-cycle of $50 \%$. The photon flux per second $(\Phi)$ is also reported in the inset.

ulating signal frequency at different pump wavelengths and powers. The dynamic behaviour in dark is also reported for comparison. We note that $M \%$ is enhanced by light, as an effect of the higher carrier concentration that takes part to the modulation process.

Moreover, we calculated the contribution to modulation brought by a visible photon flux per second $(\Phi$ [photons $\left.\left./\left(\mathrm{s} \cdot \mathrm{cm}^{2}\right)\right]\right)$. The modulation efficiency per unit pho- 


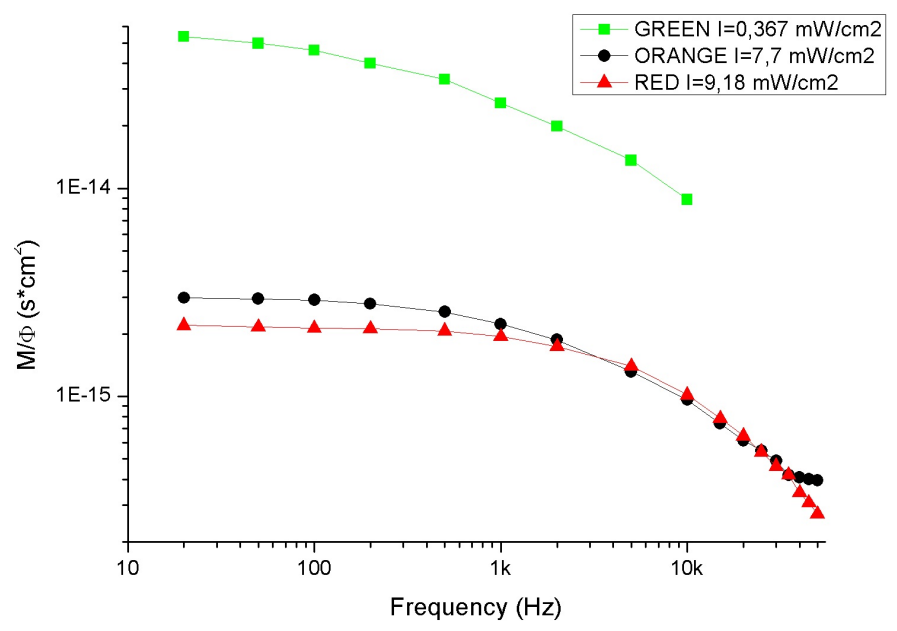

FIG. 8 Modulation efficiency: contribution to modulation brought by one photon per second.

ton flux, i.e. $M_{\%} / \Phi$, at different frequencies was calculated from the experimental modulation depth and the measured visible photon flux reported in the inset of Figure 7. In contrast to what observed above, a higher $M_{\%} / \Phi$ is achieved upon shorter wavelength pump illumination, from which we can conclude, that higher energy photons are more effective (see Figure 8).

However, $M_{\%}$ is an overall parameter, which depends not only on the change of the material optical absorption, but also on the waveguide geometry. In fact, the illumination cannot be considered uniform across the waveguide thickness, especially at pump wavelengths smaller than the absorption edge of the material. Therefore, an accurate analysis of the device dynamic behaviour requires either an analytical model of the whole assembly or recourse to numerical simulations in which the calculation of carrier concentration depth profile is coupled to the pump illumination intensity through the core waveguide thickness. Besides being more general, the latter approach is expected to give more reliable results, and is therefore used hereafter.

The following table summarises some of the simulation parameters used in ATLAS [15] in order to estimate the electron concentration profile along the $\alpha$-Si:H layers under illumination when different biasing voltages are applied across the multistack device. Refractive indices, $n$, and extinction coefficients, $k$, reported in Table 5 were experimentally measured at the listed wavelengths.

With these data, simulations return, for red pumping light $(\lambda=660 \mathrm{~nm})$, a starting (zero bias) free carrier (electrons) concentration (SFCC) at the $\alpha$-SiCN $/ \alpha-\mathrm{Si}: \mathrm{H}$ interface of $9.27 \times$ $10^{14} \mathrm{~cm}^{-3}$, and an accumulation time of $71 \mu \mathrm{s}$. The accumulation time, $t_{\mathrm{acc}}$, has been fixed at the time when the electrons reach, at the $\alpha-\mathrm{SiCN} / \alpha-\mathrm{Si}: \mathrm{H}$ interface, the concentration of $10^{18} \mathrm{~cm}^{-3}$, a reference value for which the Drude model returns a significant $\Delta \alpha$ of $6 \mathrm{~cm}^{-1}$.

In Tables 6 and 7 we show how the numerical transient behaviours match the measured ones for all pumping wavelengths considered in experiments.

\begin{tabular}{|c|c|c|c|c|}
\hline$\lambda(\mathrm{nm})$ & $\begin{array}{c}\text { Power } \\
\left(\mathrm{mW} / \mathrm{cm}^{2}\right)\end{array}$ & Layer & $\mathbf{n}$ & $\mathbf{k}$ \\
\hline \multirow{2}{*}{565} & \multirow{2}{*}{0.367} & $\alpha-\mathrm{SiCN}$ & 2.39 & 0.009 \\
\hline & & $\alpha-\mathrm{Si}: \mathrm{H}$ & 4.45 & 0.23 \\
\hline \multirow{2}{*}{612} & \multirow{2}{*}{7.14} & $\alpha-\mathrm{SiCN}$ & 2.34 & 0.005 \\
\hline & & $\alpha-\mathrm{Si}: \mathrm{H}$ & 4.3 & 0.1256 \\
\hline \multirow{2}{*}{660} & \multirow{2}{*}{9.18} & $\alpha-\operatorname{SiCN}$ & 2.32 & 0.003 \\
\hline & & $\alpha-\mathrm{Si}: \mathrm{H}$ & 4.17 & 0.0399 \\
\hline
\end{tabular}

TABLE 5 Simulations parameters, refractive index $n$ and extinction coefficient $k$ for $\alpha$-SiCN and $\alpha$-Si:H layers, used in ATLAS

\begin{tabular}{|c|c|c|c|}
\hline & \multicolumn{3}{|c|}{$\lambda(\mathrm{nm})$} \\
\hline & 565 & 612 & 660 \\
\hline $\begin{array}{l}\text { Light power } \\
\left(\mathrm{mW} / \mathrm{cm}^{2}\right)\end{array}$ & 0.367 & 7.14 & 660 \\
\hline $\begin{array}{c}\text { Stimulated } \\
\text { accumulation time } \\
(\mu \mathrm{s})\end{array}$ & 510 & 112 & 71 \\
\hline $\begin{array}{l}\text { Estimated starting } \\
\text { free carrier } \\
\text { concentration } \\
(\mathrm{SFCC}) \text { at } \\
\text { interface }\left[\mathrm{cm}^{-3}\right]\end{array}$ & $1.05 \times 10^{13}$ & $5.84 \times 10^{14}$ & $9.27 \times 10^{14}$ \\
\hline $\begin{array}{c}\text { Experimental } \\
\text { accumulation time } \\
(\mu \mathrm{s})\end{array}$ & 630 & 120 & 83 \\
\hline
\end{tabular}

TABLE 6 Comparison between calculated and measured accumulation times at different pumping wavelengths.

\begin{tabular}{|c|c|c|c|}
\hline $\begin{array}{c}\text { Light } \\
\text { power } \\
\left(\mathbf{m W} / \mathbf{c m}^{\mathbf{2}}\right)\end{array}$ & $\begin{array}{c}\text { SFCC } \\
\left(\mathbf{c m}^{-2}\right)\end{array}$ & $\begin{array}{c}\boldsymbol{t}_{\mathrm{acc}} \\
(\mathbf{s i m u l a t e d}) \\
(\boldsymbol{\mu} \mathbf{s})\end{array}$ & $\begin{array}{c}\boldsymbol{t}_{\mathrm{acc}} \\
(\mathbf{m e a s u r e d}) \\
(\boldsymbol{\mu} \mathbf{s})\end{array}$ \\
\hline $\mathbf{4 . 0 8}$ & $5.85 \times 10^{14}$ & 110 & 113 \\
\hline $\mathbf{7 . 7 5}$ & $8.48 \times 10^{14}$ & 79 & 90 \\
\hline $\mathbf{9 . 1 8}$ & $9.27 \times 10^{14}$ & 71 & 83 \\
\hline
\end{tabular}

TABLE 7 Comparison between calculated and measured accumulation times at different pumping wavelengths.

The simulated and the experimental accumulation times are very close each other, therefore we can assume that these results are a further proof of the reliability of our simulations, and in particular of the $\alpha$-Si:H model used, also in presence of illumination. It should be remarked, in fact, that the count of free carriers in $\alpha-\mathrm{Si}: \mathrm{H}$ also takes into account the carrier trapping effects by defects, typical of amorphous semiconductors $[17,18]$.

From these results, as confirmed by comparison with the experimental data, it is worth noting that the dynamic behaviour of the undoped-illuminated device is controlled by the number of available carriers in the $\alpha-\mathrm{Si}: \mathrm{H}$ layers involved in the 


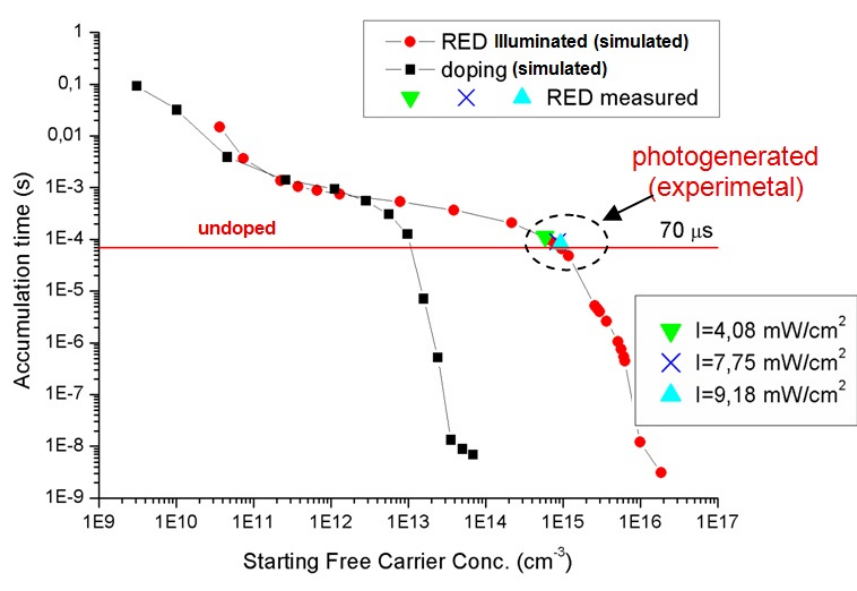

FIG. 9 Carrier accumulation time versus the zero bias free carrier concentration at the interface for doped and undoped-illuminated $\alpha$-Si:H layers.

accumulation process at each semiconductor/dielectric interface.

In the following we report a study to asses the role of native free carriers, provided through doping the $\alpha-\mathrm{Si}: \mathrm{H}$, on the device dynamics.

Figure 9 summarises the results of transient simulations performed on the same device at different doping levels of the $\alpha$ $\mathrm{Si}: H$ layers. For comparison, we also report the accumulation times for the intrinsic/red-illuminated $(\lambda=660 \mathrm{~nm})$ sample as a function of the starting $n$-concentration at the interface together with the experimental results previously reported in Table 7. As already mentioned, in this graph the "Starting Free Carrier Concentration" represents the estimated free electron density at the $\alpha$-Si:H/ $\alpha$-SiCN interface, either due to doping or to photogeneration, before the application of the electrical pulse.

Simulation results demonstrated that in order to get the same speed, a higher $n$-concentration is necessary for the intrinsicilluminated sample. In particular, for the given accumulation time, the photogenerated carriers at the interface must be approximately 100 times more than the native majority carriers provided by doping. Moreover, a majority carrier concentration of $10^{14} \mathrm{~cm}^{-3}$ should allow modulation times of the order of $1 \mathrm{~ns}$ allowing to design a device operating at higher frequency bandwidth at expense, however, of higher absorption optical losses.

From resistivity measurements we calculated that the free electron concentration in our device (undoped) is close to $10^{10} \mathrm{~cm}^{-3}$, which returns an accumulation speed of $3 \times 10^{-2} \mathrm{~s}$, in good agreement with the experimental results of Figure 5.

As already mentioned, from electrical simulation results, we noted that the electron concentration profiles along the $\alpha$-Si:H layers are rather different for the illuminated sample with respect to the correspondent doped device, since the first has a higher interface carrier concentration, but a lower internal profile.
However, if we calculate the integral of the two concentration profiles we see that they tend to be very close each other. In fact, while in the intrinsic-illuminated sample the excess carriers are mostly provided by absorbed photons of visible radiation continuously penetrating from the top, for the doped sample they are rather uniformly present in the overall $\alpha-\mathrm{Si}: \mathrm{H}$ thickness (or provided by thermal generation, which is however rather weak).

An additional proof of the role of the integral carriers on speed comes from simulation results listed in Table 8, where, for devices with the same accumulation times, $t_{\mathrm{acc}}$, (doped or under red illumination), we report the Starting Free Carrier Concentration at the interface (SFCC) and the Starting Integral Free Carrier Concentration (ISFCC) along the $\alpha$-Si:H layer. We note that the ISFFC's are much closer each other than the SFFC's, meaning that the overall charge count is in fact determining the device speed. The ISFFC's values get closer and closer each other for shorter $t_{\mathrm{acc}}$.

In addition, it is worth noting that, according to simulations, the illumination intensity required to get 12 ns times is approximately two order of magnitude higher than the one used in our experiments. Beside the fact these levels of illumination are not easily feasible, they would certainly induce additional effects, such as a temperature increase. Moreover, photogeneration tends to saturate at those levels, and the absorption coefficient is certainly different from that at low photon fluxes.

In parallel, the required doping should be increased by a factor of two only, in order to get the same short times.

\section{CONCLUSION}

A multi-stack structure, based on the CMOS-compatible technology of amorphous silicon, has been explored for enhancing electro-optical modulation effects in integrated, waveguide embedded, photonic devices.

The waveguiding structure, consisting of six $\alpha-\mathrm{Si}: \mathrm{H} / \alpha-\mathrm{SiCN}$ stacks deposited by PECVD on a c-Si substrate, were realized and characterized. It showed propagation losses lower than $2.3 \mathrm{~cm}^{-1}$ at the wavelength of $1.55 \mu \mathrm{m}$. The application of an external electric field across the structure produces carrier accumulation at the intermediate semiconductor/insulator interfaces, which is in turn responsible of an enhanced optical absorption. We measured a $\Delta \alpha_{\text {eff }}$ of $0.75 \mathrm{~cm}^{-1}$ when an electric field of $0.39 \mathrm{MV} / \mathrm{cm}$ is applied across each $\alpha-\mathrm{SiCN}$ dielectric thin film.

The dynamic behaviour of the device was studied, theoretically and experimentally, by using as pump radiation three visible light emitting diodes (LEDs).

Results have shown the impact of available free carriers in the $\alpha$-Si:H layers provided by photogeneration (for undoped device) or by doping the amorphous semiconductor.

Compared to illumination, small variations of doping have a much stronger impact on transient response. 


\begin{tabular}{|c|c|c|c|c|c|}
\hline \multirow[t]{2}{*}{$t_{\mathrm{acc}}[\mathrm{s}]$ (simulation) } & \multicolumn{3}{|c|}{$\begin{array}{c}\text { Red } \\
(\lambda=600 \mathrm{~nm})\end{array}$} & \multicolumn{2}{|c|}{ Doping } \\
\hline & Intensity $\left[\mathrm{mW} / \mathrm{cm}^{2}\right]$ & $\mathrm{SFCC}\left[\mathrm{cm}^{-3}\right]$ & ISFCC $\left[\mathrm{cm}^{-2}\right]$ & $\mathrm{SFCC}\left[\mathrm{cm}^{-3}\right]$ & ISFCC $\left[\mathrm{cm}^{-2}\right]$ \\
\hline $3.67 \times 10^{-3}$ & $1 \times 10^{-3}$ & $7.4 \times 10^{10}$ & $2.6 \times 10^{7}$ & $4.7 \times 10^{10}$ & $2.0 \times 10^{8}$ \\
\hline $2.08 \times 10^{-4}$ & 1 & $2.2 \times 10^{14}$ & $1.8 \times 10^{10}$ & $7.5 \times 10^{12}$ & $1.3 \times 10^{11}$ \\
\hline $4.74 \times 10^{5}$ (close to exp. value) & 15 & $1.2 \times 10^{15}$ & $4.2 \times 10^{10}$ & $1.3 \times 10^{13}$ & $1.8 \times 10^{11}$ \\
\hline $7.55 \times 10^{-7}$ & 300 & $5.7 \times 10^{15}$ & $8.3 \times 10^{10}$ & $2.3 \times 10^{13}$ & $2.6 \times 10^{11}$ \\
\hline $1.21 \times 10^{-8}$ & 1000 & $9.9 \times 10^{15}$ & $1.1 \times 10^{11}$ & $3.8 \times 10^{13}$ & $3.4 \times 10^{11}$ \\
\hline
\end{tabular}

TABLE 8 Estimated starting free carrier concentration at interface and starting integral free carrier concentration for doped $\alpha$-Si:H and under red illumination at fixed accumulation times.

We found a good agreement between simulated and experimental data, both for static and dynamic operations.

\section{ACKNOWLEDGEMENTS}

The authors wish to thank C. Summonte (IMM-CNR Bologna, Italy), G. T. Reed and W. Headley (University of Surrey, Guildford, UK) for helps in some experiments and for stimulating discussions.

IMM-CNR, Units of Bologna and Napoli (Italy), where the waveguide was fabricated and where the optical simulations were performed, are gratefully acknowledged.

This work was partially developed under VII FP of the EC ICT project "HELIOS" - Grant Agreement Number 224312.

\section{References}

[1] G. Cocorullo, F. G. Della Corte, and I. Rendina, "Amorphous silicon waveguides and light modulators for integrated photonics realized by low-temperature plasma-enhanced chemical-vapor deposition" Opt. Lett. 21, 2002-2004 (1996).

[2] G. Cocorullo, F. G. Della Corte, R. De Rosa, I. Rendina, A. Rubino, and E. Terzini, "Amorphous silicon-based guided-wave passive and active devices for silicon integrated optoelectronics" IEEE J. Sel. Top. Quant. 4, 997-1002 (1998).

[3] 0. B. Usev, A. M. Kuznetsov, E. I. Terukov, M. S. Bresler, V. K. Kudoyarova, I. N. Yassievich, B. P. Zakharchenya, and W. Fuhs, "Room-temperature electroluminescence of erbium-doped amorphous hydrogenated silicon" Appl. Phys. Lett. 70, 240-242 (1997).

[4] M. Okamura, and S. Suzuki, "Infrared photodetection using $\alpha \mathrm{Si}: \mathrm{H}$ photodiode" IEEE Photonic. Tech. L. 6, 412-414 (1994).

[5] C. Summonte, F. G. Della Corte, M. A. Nigro, and A. Desalvo "Photoinduced absorption in B-doped hydrogenated amorphous silicon alloys applied to all-optical modulators" J. Appl. Phys. 103, 023107 (2008).

[6] W. B. Jackson, N. M. Amer, A. C. Boccara, and D. Fournier, "Photothermal deflection spectroscopy and detection" Appl. Opt. 20, 1333-1344 (1981).

[7] M. Zelikson, K. Weiser, A. Chack, and J. Kanicki, "Direct determination of the quadratic electro-optic coefficient in an $\alpha$-Si:H based waveguide" J. Non-Cryst. Solids 198, 107-110 (1996).

[8] F. C. Della Corte, S. Rao, M. A. Nigro, F. Suriano, and C. Summonte, "Electro-optically induced absorption in $\alpha \mathrm{Si}: \mathrm{H} / \alpha$-SiCN waveguiding multistacks" Opt. Express 16, 7540-7550 (2008).

[9] RSoft Photonics CAD Layout User Guide (RSoft Design Group, Ossining).

[10] C. A. Barrios, and M. Lipson, "Modeling and analysis of high-speed electro-optic modulation in high confinement silicon waveguides using metal-oxide-semiconductor configuration" J. Appl. Phys. 96, 6008-6015, (2004).

[11] A. Liu, R. Jones, L. Liao, D. Samara-Rubio, D. Rubin, 0. Cohen, R. Nicolaescu, and M. Paniccia, "A high speed silicon optical modulator based on a metal-oxide-semiconductor capacitor" Nature 427, 615-618, (2004).

[12] G. Lavareda, C. Nunes de Carvalho, E. Fortunato, A. Amaral, and A. R. Ramos, "Properties of $\alpha$-Si:H TFTs using silicon carbonitride as dielectric" J. Non-Cryst. Solids 338, 797-801 (2004).

[13] P. M. Fauchet, D. Hulin, R. Vanderhaghen, A. Mourchid, and W. L. Nighan Jr., "The properties of free carriers in amorphous silicon" J. Non-Cryst. Solids 141, 76-87 (1992).

[14] C. A. Barrios, "Electrooptic modulation of multisilicon-on-insulator photonic wires" J. Lightwave Technol. 24, 2146-2155 (2006).

[15] ATLAS device simulation software user's manual (SILVACO Int., Santa Clara, 2005).

[16] R. A. Street, Hydrogenated Amorphous Silicon (Cambridge University Press, Cambridge, 1991).

[17] J. Singh, "Effective mass of charge carriers in amorphous semiconductors and its applications" J. Non-Cryst. Solids 120, 295-300 (1973).

[18] F. G. Della Corte, A. Rubino, and G. Cocorullo, "Simulation study and realisation of an $\alpha$-Si:H emitter on GaAs" Solid State Electron. 42, 1819-1825 (1998).

[19] G. Cancellieri, and U. Ravaioli, Measurements of Optical Fibers and Devices: Theory and Experiments (Dedham, Artech House, 1984).

[20] G. V. Treyz, P. G. May, and J. M. Halbout, "Silicon optical modulators a $1.3 \mu \mathrm{m}$ based on free carrier absorption" IEEE Electr. Device L. 12, 276-278 (1991).

[21] A. Sciuto, S. Libertino, S. Coffa, and G. Coppola, "Miniaturizable Si-based electro-optical modulator working at $1.5 \mu \mathrm{m}$ " Appl. Phys. Lett. 86, 20115 (2005).

[22] T. Tabei, T. Hirata, K. Kajikawa, and H. Sunami, "Potentiality of silicon optical modulator based on free-carrier absorption" in IEEE International Electron Devices Meeting 2007, 1023-1026 (IEEE, Washington D.C., 2007). 